\title{
White matter deficits in cocaine use disorder: convergent evidence from in vivo diffusion tensor imaging and ex vivo proteomic analysis
}

Lucca Pizzato Tondo (1)', Thiago Wendt Viola', Gabriel R. Fries (1)², Bruno Kluwe-Schiavon',

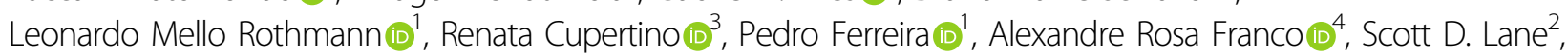
Laura Stertz ${ }^{2}$, Zhongming Zhao $\mathbb{0}^{5}$, Ruifeng Hu $\mathbb{0}^{5}$, Thomas Meyer ${ }^{2}$, Joy M. Schmitz ${ }^{2}$, Consuelo Walss-Bass ${ }^{2}$ and Rodrigo Grassi-Oliveira (1) ${ }^{1,6}$

\begin{abstract}
White matter (WM) abnormalities in patients with cocaine use disorder (CUD) have been studied; however, the reported effects on the human brain are heterogenous and most results have been obtained from male participants. In addition, biological data supporting the imaging findings and revealing possible mechanisms underlying the neurotoxic effects of chronic cocaine use (CU) on WM are largely restricted to animal studies. To evaluate the neurotoxic effects of $\mathrm{CU}$ in the WM, we performed an in vivo diffusion tensor imaging assessment of male and female cocaine users $(n=75)$ and healthy controls $(\mathrm{HC})(n=58)$. Moreover, we performed an ex vivo large-scale proteomic analysis using liquid chromatography-tandem mass spectrometry in postmortem brains of patients with CUD $(n=8)$ and $\mathrm{HC}(n=12)$. Compared with the HC, the CUD group showed significant reductions in global fractional anisotropy (FA) $(p<0.001)$, and an increase in global mean (MD) and radial diffusion (RD) (both $p<0.001)$. The results revealed that FA, $R D$, and $M D$ alterations in the CUD group were widespread along the major WM tracts, after analysis using the tract-based special statistics approach. Global FA was negatively associated with years of CU $(p=0.0421)$ and female sex $(p<0.001)$, but not with years of alcohol or nicotine use. Concerning the fibers connecting the left to the right prefrontal cortex, Brodmann area 9 (BA9), the CUD group presented lower FA $(p=0.006)$ and higher RD $(p<0.001)$ values compared with the $\mathrm{HC}$ group. A negative association between the duration of $C U$ in life and FA values in this tract was also observed $(p=0.019)$. Proteomics analyses in BA9 found 11 proteins differentially expressed between cocaine users and controls. Among these, were proteins related to myelination and neuroinflammation. In summary, we demonstrate convergent evidence from in vivo diffusion tensor imaging and ex vivo proteomics analysis of WM disruption in CUD.
\end{abstract}

\section{Introduction}

Cocaine use disorder (CUD) is a chronic disease characterized by compulsive drug use with negative

\footnotetext{
Correspondence: Rodrigo Grassi-Oliveira (rogo@clin.au.dk)

${ }^{1}$ Developmental Cognitive Neuroscience Lab (DCNL), Brain Institute, Pontifical Catholic University of Rio Grande do Sul (PUCRS), Porto Alegre, Brazil

${ }^{2}$ Louis A. Faillace, MD, Department of Psychiatry and Behavioral Sciences, University of Texas Health Science Center at Houston, Houston, TX, USA

Full list of author information is available at the end of the article
}

psychosocial consequences, affecting $\sim 0.5-3 \%$ of the adult population worldwide ${ }^{1,2}$. It is a severe, complex, and debilitating illness leading to higher mortality rates and several daily life disturbances ${ }^{3-5}$. In the brain, CUD is associated with decreased dopamine signaling and widespread structural and functional alterations ${ }^{6}$.

Although most neuroimaging studies have examined the chronic cocaine use (CU) effects on specific gray matter brain regions, several recent investigations have 
focused on revealing the white matter (WM) signature of $\mathrm{CUD}^{7}$. Diffusion-weighted imaging (DWI) is a magnetic resonance sequence that captures water diffusion properties in the brain and has been frequently used in studies that determined the fibers and tracts that are progressively deteriorated because of repeated cocaine consumption. In addition, diffusion tensor imaging (DTI) is a widely used method to model the DWI signal ${ }^{8}$, which allows the in vivo assessment of WM microstructural characteristics through the analysis of eigenvalues and eigenvectors. These eigenvectors are used to derive the four main metrics analyzed in DTI studies: fractional anisotropy (FA), axial diffusion (AD), radial diffusion (RD), and mean diffusion (MD). Generally, properly myelinated fibers present high FA and low RD and MD values ${ }^{9}$. FA changes can reflect the coherence of fibers, microglia, axon packing, density, myelination, or inflammation ${ }^{10}$, and its decreased signal has been associated with many diseases affecting the WM integrity ${ }^{11}$.

Previous studies have supported that CUD is associated with decreased FA signal ${ }^{12-15}$. A recent meta-analysis of data obtained from DTI studies that included patients with stimulant use disorder revealed a small-to-moderate group-effect in reducing the FA values; conversely, a subanalysis focusing on patients with CUD showed greater heterogeneity of findings and effect sizes ${ }^{16}$. Another metaanalysis including only data from tract-based special statistics (TBSS) studies for CUD revealed a decreased FA in the genu of the corpus callosum and a tenuous effect of pre-imaging abstinence on FA values ${ }^{17}$. Regarding the clinical associations, a study has found that the decrease in FA values is correlated with the years of $\mathrm{CU}^{18}$. Other studies have shown a positive association between the FA values at treatment onset and the abstinence duration during cocaine addiction treatment ${ }^{15,19}$. Moreover, cognitive impairments in decision-making ${ }^{20}$ and working memory ${ }^{21}$ have been associated with altered DTI metrics among cocaine users.

However, the heterogeneous distribution of DTI findings in patients with CUD highlights the challenges and limitations of the currently available research. First, most studies have been conducted including mainly male participants, in which the CUD group is generally composed of $<40$ individuals ${ }^{16}$. This may result in difficulties in obtaining precise DTI comparisons between users and non-users beyond the effect sizes of major callosal tracts and limited evidence regarding the female cocaine users. In addition, although some studies have documented clinical associations with DTI metrics, small sample sizes generate additional obstacles for such analyses, especially when accounting for covariate effects (e.g., age and sex). Second, $>60 \%$ of individuals with CUD report chronic alcohol use consumption ${ }^{22-25}$. The high comorbidity profile suggests that the evaluation of samples obtained from polysubstance users is important, especially for the generalization of the findings to the general population ${ }^{26}$. Third, biological investigations that support the imaging findings and reveal possible mechanisms underlying the chronic CU neurotoxic effects on the WM are largely restricted to animal studies. Some of these studies have suggested that the reduced myelin-related protein levels in the brain may correlate with the decreased FA signal in the corpus callosum ${ }^{27,28}$, despite human studies using postmortem brain tissues have identified several neurobiological changes in chronic cocaine users ${ }^{29}$, particularly decreased myelin-related gene expression, suggesting an altered myelin structure and integrity ${ }^{30}$. DWI measures rely on multiple factors such as axonal coherence, membrane permeability, and myelination ${ }^{10}$, and their alteration cannot be interpreted, by itself, as conclusive evidence of WM impairment: proteomic and histological findings corroborating neuroimage data are essential.

Considering these limitations, we performed a comprehensive study combining two distinct approaches that evaluated the CUD neurotoxic effects in brain WM. First, we compared the DTI metrics between individuals with and without CUD, including male and female individuals, using two complementary post-processing techniques: TBSS and deterministic tractography ${ }^{31}$. Moreover, we recruited a larger number of participants than usual for DTI studies. First, we focused on crack-cocainedependent individuals, most of whom report concurrent tobacco and alcohol use, to meaningfully capture a more representative sample of polysubstance users ${ }^{32,33}$. Then, we assessed the protein expression levels in the brain of individuals with and without CUD to determine whether alterations in pathways related to WM integrity in the human brain are affected by chronic CU. To that end, we performed a large-scale proteomic analysis in postmortem brains. We hypothesized that an investigation combining in vivo imaging and ex vivo biochemical approaches would extend previous findings and better explain the biological mechanisms associated with WM morphological deficits in patients with CUD.

\section{Methods}

The in vivo imaging examinations were performed in patients with CUD during the acute abstinence phase of smoked cocaine (crack-cocaine) detoxification treatment. The ex vivo postmortem brain study was performed using brain tissues collected from individuals whose families donated their brains to the UTHealth Brain Collection for Research in Psychiatric Disorders (Houston, TX, USA). The ethics committees of the institutions involved (PUCRS and UTHealth) in both studies approved the research protocol. This study was conducted in accordance with the Declaration of Helsinki. All participants (in vivo study) or their families (ex vivo study) provided 
written informed consent after receiving a complete description of the study.

\section{In vivo neuroimaging sample}

The participants were 75 patients with CUD who were recruited during inpatient detoxification and drug rehabilitation treatment in southern Brazil. The treatment consisted of psychoeducation and support groups, moderate physical activity, a balanced diet $(2200 \mathrm{Kcal} /$ day $)$, nursing care, and psychological and medical treatments. The patients remained in a controlled environment without access to alcohol, illicit drugs, and tobacco. Prior to neuroimaging assessment, we collected urine samples from the participants to test for cocaine, benzodiazepines, cannabis, amphetamine, and opiates using an instant urine drug test (EDOAP754, Easy Healthcare Corporation, Burr Ridge, IL, USA). The inclusion criteria for the cocaine group were as follows: (1) diagnosis of CUD according to the Structured Clinical Interview for DSMIV Axis I Disorders (SCID-IV) ${ }^{34}$; (2) IQ $>80$ based on the Wechsler Abbreviated Scale of Intelligence- $\mathrm{II}^{35}$; (3) age, 18-45 years; (4) right-handed; (5) a positive urine test for cocaine at admission; and (6) reporting cocaine as the preferred drug.

All participants had all medications suspended and tested negative for cocaine, cannabis, amphetamines, opioids, and benzodiazepines on the day of the magnetic resonance imaging (MRI) examination, which occurred 2 weeks after admission to the detoxification unit.

Fifty-eight healthy and unmedicated control participants (healthy controls; $\mathrm{HC}$ ) with a similar age and socioeconomic background were included by convenience sampling (advertising). Eligible participants were those with: (1) a negative history of use of any illicit drug; (2) no psychiatric diagnosis except for nicotine use according to the SCID-IV axis I.

The exclusion criteria were as follows: (1) positive drug urine test on the day of MRI examination; (2) restrictions related to MRI procedures; (3) current psychotic symptoms; (4) neurologic disorders; and (5) presence of human immunodeficiency virus or syphilis.

The demographic characteristics of the participants are presented in Table 1.

\section{Severity of drug use}

To better characterize the CUD sample and to investigate the severity of drug use, the participants completed the Addiction Severity Index (ASI-6) ${ }^{36}$. The severity score for each domain is affected by the following conditions: drug use, family-related issues, alcohol consumption, psychiatric issues, medical problems, legal issues, financial problems, lack of social support, and social problems. They standardized that the $\mathrm{T}$ scores have a mean of 50 and a standard deviation of 10 , theoretically ranging from
0 to 100 . Higher scores indicate greater problem severity. The number of days of substance consumption prior to treatment enrollment (last 30 days) and the years of regular substance use (cannabis, tobacco, alcohol, and $\mathrm{CU}$ at least 3 days per week) were inquired ${ }^{24}$.

\section{Neuroimaging}

See detailed information in Supplemental Methods.

T1-weighted anatomic and DWI images were acquired on a 3 T Signa GE scanner (GE Healthcare, Chicago, IL, USA) at the Brain Institute of Rio Grande do Sul, Brazil. FMRIB Software Library (FSL; Oxford, UK) version 6.0 was used to preprocess and extract $F A, M D, A D$, and RD maps from the DWI data set. Voxelwise statistical analysis of the FA data were performed using TBSS' ${ }^{37}$ pipeline: brain masks were created with bet, eddy-current and head-movement distortions were corrected using affine registration of all gradient volumes with the $b=0$ volume with $e d d y^{37}$, and estimation of DWI measures with dtifit. Between-group comparison and within crack-cocaine group analysis was performed FSL's general linear model software randomize. The results were corrected with a threshold-free cluster enhancement approach (TFCE) ${ }^{38}$ and controlled for age, sex, and years of education. Whole-brain metrics were also analyzed. Considering TBSS' methodological limitations, especially the intraparticipant variability for minor tracts and anatomical specificity of tracts within the skeleton ${ }^{39}$, we also conducted a tractography-based analysis with DSI Studio (http://dsistudio.labsolver.org/) to investigate the connectivity of the cortical regions whose specific tracts passed through the affected WM regions in TBSS. The diffusion data for each participant were reconstructed in the corresponding $\mathrm{T} 1$ image and, therefore, the fiber tracking could be performed in the participants' native space. DTI metrics for the tracts connecting Brodmann area 9 (BA9) with Brodmann area 8 (BA8) and connecting left and right BA9 were extracted and analyzed. Neuroimaging data have been deposited to the OpenNeuro repository (https://openneuro.org/datasets/ ds003599/versions/1.0.0).

\section{Ex vivo postmortem brain sample}

Brain tissue from 20 participants was collected postmortem from individuals diagnosed with CUD $(n=8)$ and from $\mathrm{HC}$ individuals without any known psychiatric disorders $(n=12)$. The samples were matched for age and postmortem interval (PMI). As part of the clinical information, the medical records were obtained and a detailed psychological autopsy was performed on all participants by interviewing the next-of-kin. Information regarding the psychiatric clinical phenotypes (evidence of depression, mania, and psychosis) stressful life events, age of drug use onset, types of drugs used, smoking and drinking history, and any co-morbidities, was obtained. 
Table 1 Sociodemographic and clinical characteristics of the samples.

\begin{tabular}{|c|c|c|c|c|}
\hline & \multicolumn{4}{|l|}{ Neuroimaging sample } \\
\hline & CUD $(n=75)($ mean $/ \%, S D / N)$ & Controls $(n=58)($ mean $/ \%, S D / N)$ & Statistics $(d f)$ & $p$ \\
\hline \multicolumn{5}{|l|}{ Sociodemographic } \\
\hline Age (years) & $32.86(7.08)$ & $27.98(7.12)$ & $t(131)=-3.93$ & $>0.0001$ \\
\hline Sex (male) & $49.3(37)$ & $36.2(21)$ & $x^{2}(1)=2.29$ & 0.130 \\
\hline Ethnicity (whites) & $44.6(33)$ & $65.5(36)$ & $x^{2}(3)=5.95$ & 0.114 \\
\hline Education (years) & $7.39(2.75)$ & $11.33(1.23)$ & $W=3768$ & $>0.0001$ \\
\hline \multicolumn{5}{|l|}{ ASI-6 (standardized T scores) } \\
\hline Drugs & $52.08(7.13)$ & - & & \\
\hline Family/child & $55.96(10.01)$ & - & & \\
\hline Alcohol & $50.34(8.85)$ & - & & \\
\hline Psychiatric status & $50.50(7.74)$ & - & & \\
\hline Medical & $43.42(8.11)$ & - & & \\
\hline Legal status & $49.89(5.71)$ & - & & \\
\hline Employment & $38.94(4.29)$ & - & & \\
\hline Family/social support & $50.94(12.15)$ & - & & \\
\hline Family/social problems & $52.41(8.96)$ & - & & \\
\hline \multicolumn{5}{|l|}{ SCID-I (SUD-current) } \\
\hline Cannabis & $29.3(22)$ & - & & \\
\hline Snorted cocaine & $14.7(11)$ & - & & \\
\hline Inhalant & $1.3(1)$ & - & & \\
\hline Tobacco & $73.3(55)$ & $5.1(3)$ & $x^{2}(1)=61.79$ & $>0.0001$ \\
\hline Alcohol & $29.3(22)$ & - & & \\
\hline \multicolumn{5}{|l|}{ Years of regular drug use-mean } \\
\hline \multicolumn{5}{|l|}{$(S D)$} \\
\hline Tobacco & $5.82(9.10)$ & $3.90(8.8)$ & $W=1102$ & $>0.0001$ \\
\hline Alcohol & $6.64(7.66)$ & $0.25(0.94)$ & - & - \\
\hline Cannabis & $7.93(7.63)$ & $0.10(0.45)$ & - & - \\
\hline \multirow[t]{3}{*}{ Cocaine } & $8.18(6.38)$ & $0(0)$ & - & - \\
\hline & \multicolumn{4}{|l|}{ Proteomics sample } \\
\hline & CUD $(n=8)($ mean/\%, SD/N $)$ & Controls $(n=12)($ mean/\%, SD/N) & Statistics $(d f)$ & $p$ \\
\hline \multicolumn{5}{|l|}{ Sociodemographic } \\
\hline Age (years) & $45.7(9.72)$ & $51.7(13.81)$ & $W=61.50$ & 0.082 \\
\hline Sex (male) & $62.5(5)$ & $83.3(10)$ & $x^{2}(1)=1.11$ & 0.292 \\
\hline Ethnicity (whites) & $25(2)$ & $58.3(7)$ & Fisher exact & 0.197 \\
\hline Post-mortem intervals (hours) & $24.1(8)$ & $29.4(8.4)$ & $W=67$ & 0.190 \\
\hline
\end{tabular}

$S D$ standard deviation, $t$ student $t$ test statistics; $X^{2}$ chi-squared, $W$ Wilcoxon rank-sum test statistics. Significant values in bold.

A CUD diagnosis was confirmed based on the psychological autopsy, detailed medical records, and review of all relevant case information by three psychiatrists at a consensus meeting. The cause of death was obtained from the medical examiner's report and toxicological findings after death. 


\section{Proteomics analysis}

Frozen brain tissues $(50 \mathrm{~g})$ obtained from the dorsolateral prefrontal cortex BA9 were lysed in a radioimmunoprecipitation assay buffer and the extracted proteins were reduced, alkylated, de-lipidated, and digested with trypsin. Peptide concentrations were determined by Nanodrop (Thermo Fisher Scientific, Waltham, MA, USA) measurements at A280 nm. Nanoflow liquid chromatography-tandem mass spectrometry (LC-MS/ MS) was performed using a nano-LC chromatography system, coupled online to a mass spectrometer through a nanospray ion source. LC-MS/MS data were acquired using XCalibur (Thermo Fisher Scientific) and the raw mass spectrometry data files were processed using MaxQuant (Max-Planck Institute of Biochemistry, Planegg, Germany). Peptide identifications were accepted in cases where they could be established at a probability $>95.0 \%$. MS/MS spectra were searched against the Swiss-Prot human database. In total, 4584 unique proteins were identified in all the samples.

\section{Statistical analysis}

Statistical analyses were performed using the Statistical Package for the Social Sciences (SPSS version 26; IBM Corp., Armonk, NY, USA) and R software (version 3.6.1; R Foundation for Statistical Computing, Vienna, Austria). Regarding the demographic, clinical, and substancerelated variables, the frequency data were analyzed using the Pearson's chi-squared test. Group data were compared using Student's $t$ tests. When the data were non-normally distributed we used the Wilcoxon rank-sum test.

Regarding TBSS analysis with randomize algorithm, a $p$ value $<0.05$ after TFCE was considered significant. Given the known effects of age, sex, and years of education on $\mathrm{FA}^{40-42}$, these variables were added as covariates in both analyses along with head motion (estimated by eddy output). Within-group analysis also included the duration (years) of cocaine, nicotine, and alcohol use, as covariates. We used analysis of covariance (ANCOVA) to compare the global FA between the groups and we performed a linear regression analysis for within-group substance use associations.

Given that the TBSS cluster analysis revealed a major effect of years of crack-cocaine consumption on FA in the genu of the corpus callosum, and that previous studies demonstrated a main effect of cocaine in this anterior region ${ }^{17}$, we further explored this association by focusing on specific fibers of the frontal lobe. The anterior corpus callosum contains fibers responsible for prefrontal interhemispheric connectivity ${ }^{43,44}$. As structural abnormalities in the prefrontal cortex have been observed in patients with CUD ${ }^{45,46}$, we additionally selected the BA9 and BA8, as our main regions of interest (ROIs) for tractography ${ }^{47}$. These ROIs were extracted and projected into each participant's surface through FreeSurfer software utilities. We reconstructed and analyzed the fibers connecting BA9 to BA8 in each hemisphere and fibers connecting the left to the right BA9.

Concerning the analysis of the WM bundles derived from tractography, we used ANCOVA to compare the groups considering sex and the duration of $\mathrm{CU}$ in life (years of CU/age).

For the proteomics analysis, the data were normalized and imputed. Moreover, differential expression analyses were performed in patients with CUD and HCs using protein-wise linear models combined with empirical Bayesian statistics implemented in the differential enrichment analysis of Proteomics data package and the limma package (R software), controlling for age, sex, PMI, and $\mathrm{pH} ; p$ values $<0.05$ and fold changes $(\mathrm{FC})>1.5$ were considered significant. Gene set enrichment analysis was performed using Webgestalt software (access date: 20 April 2019). We required gene sets of 5-1000 genes per set to avoid specific or general annotations. We assessed pathways in two categories: GO-Cellular Component and KEGG pathways. Within each category, the terms/pathways with $\mathrm{FDR}<0.05$ were considered significant.

\section{Results \\ Demographic and clinical characteristics}

The cause of death was obtained from the medical examiner's report and toxicological findings after death. The cause of death for individuals with CUD was cocaine overdose $(n=7)$ and cardiovascular disease $(n=1)$. The cause of death of the HCs was cardiovascular disease $(n=10)$ and pulmonary embolism $(n=2)$. The sociodemographic and clinical variables of the participants regarding the neuroimaging investigation are presented in Table 1 . The data regarding the postmortem brain sample are presented in Table 1. Interestingly, the individuals in the CUD group had significantly higher age and less years of education compared to those in the $\mathrm{HC}$ group. Most of our samples consisted of severe cocaine users according with ASI-6. In addition, at least $1 / 3$ of them could be classified as polysubstance users, as they met the criteria for cannabis, alcohol, or tobacco use disorders. There were no other statistically significant sociodemographic differences between the groups.

\section{TBSS comparison between the CUD and HC groups}

Compared with the HC group, the CUD group showed a significant decrease in the global FA (COD group: mean, $0.449 \pm 0.015$; HC group: mean, $0.459 \pm 0.015 ; p<0.001$ ) and an increase in the global MD (COD group: mean, $0.00096 \pm 3 \mathrm{e}-05$; HC group: mean, $0.00093 \pm 3 \mathrm{e}-05 ; p<$ 0.001 ) and RD (COD group: mean, $0.00070 \pm 3 e-05$; HC group: mean, $0.00068 \pm 3 \mathrm{e}-05 ; p<0.001)$. The obtained results revealed that $\mathrm{FA}, \mathrm{RD}$, and $\mathrm{MD}$ alterations in the 


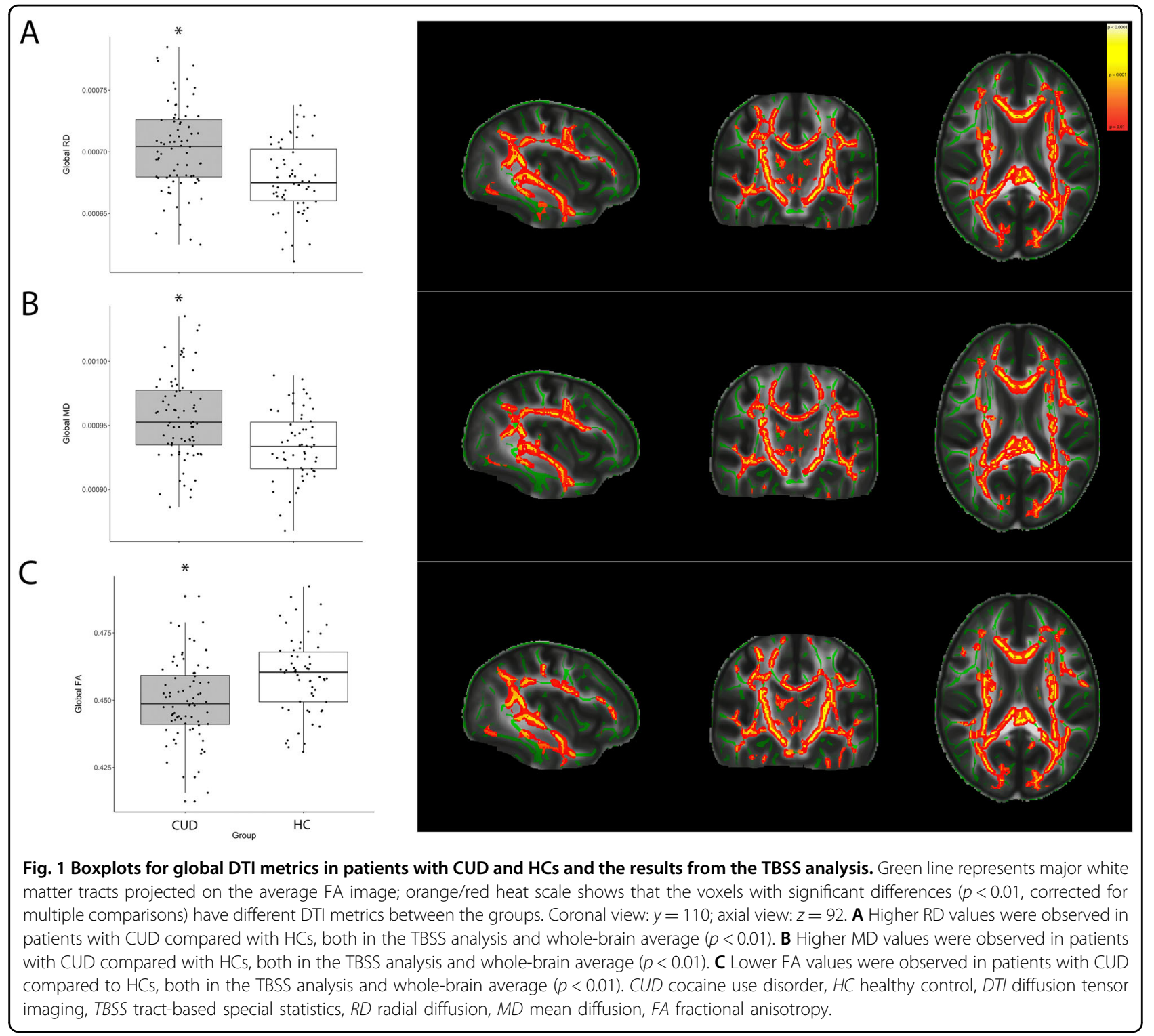

CUD group were widespread along the major WM tracts, as revealed by TBSS analysis (Fig. 1). Information on the percentage of significant voxels for each WM region can be found in Supplementary Table 1. There was also an independent effect of sex, with women having lower global FA and AD (both $p<0.001$ ) values than men (TBSS showed a widespread difference in both metrics).

\section{TBSS within the CUD group}

We performed an additional TBSS analysis within the CUD group to further characterize the alterations in DTI metrics. The results $(F(6,68)=2.693 ; p<0.003$; adjusted $\left.R^{2}=0.137\right)$ showed that the global FA was negatively associated with the years of $\mathrm{CU}(t=-2.078 ; p=0.0421)$ and female sex $(t=-3.553 ; p<0.001)$, but not with the years of alcohol or nicotine use. Cluster identification provided by TFCE revealed 12 FA clusters along multiple major WM tracks (including the cingulum bundle, corpus callosum, and uncinate fasciculus), in which the FA values were negatively correlated with years of CU (Fig. 2A). Most of these clusters were also negatively correlated with the female sex. One major cluster (Fig. 2B) comprising the genu and body of the corpus callosum (1505 voxels, center of mass: $x=94, y=155, z=81$ ) showed an exclusive association with the years of $\mathrm{CU}(t=-3.145 ; p<0.001)$ and no other variable $(F(6,68)=5.454 ; p=0.001$; adjusted $\left.R^{2}=0.265\right)$.

The global RD showed no significant correlation with any studied variable, but two clusters (Fig. 3A, B) had RD values positively associated with the years of CU. The cluster displayed in Fig. 3A was related $(F(6,68)=$ 4.428724; $p<0.001$; adjusted $\left.R^{2}=0.232\right)$ with years of 

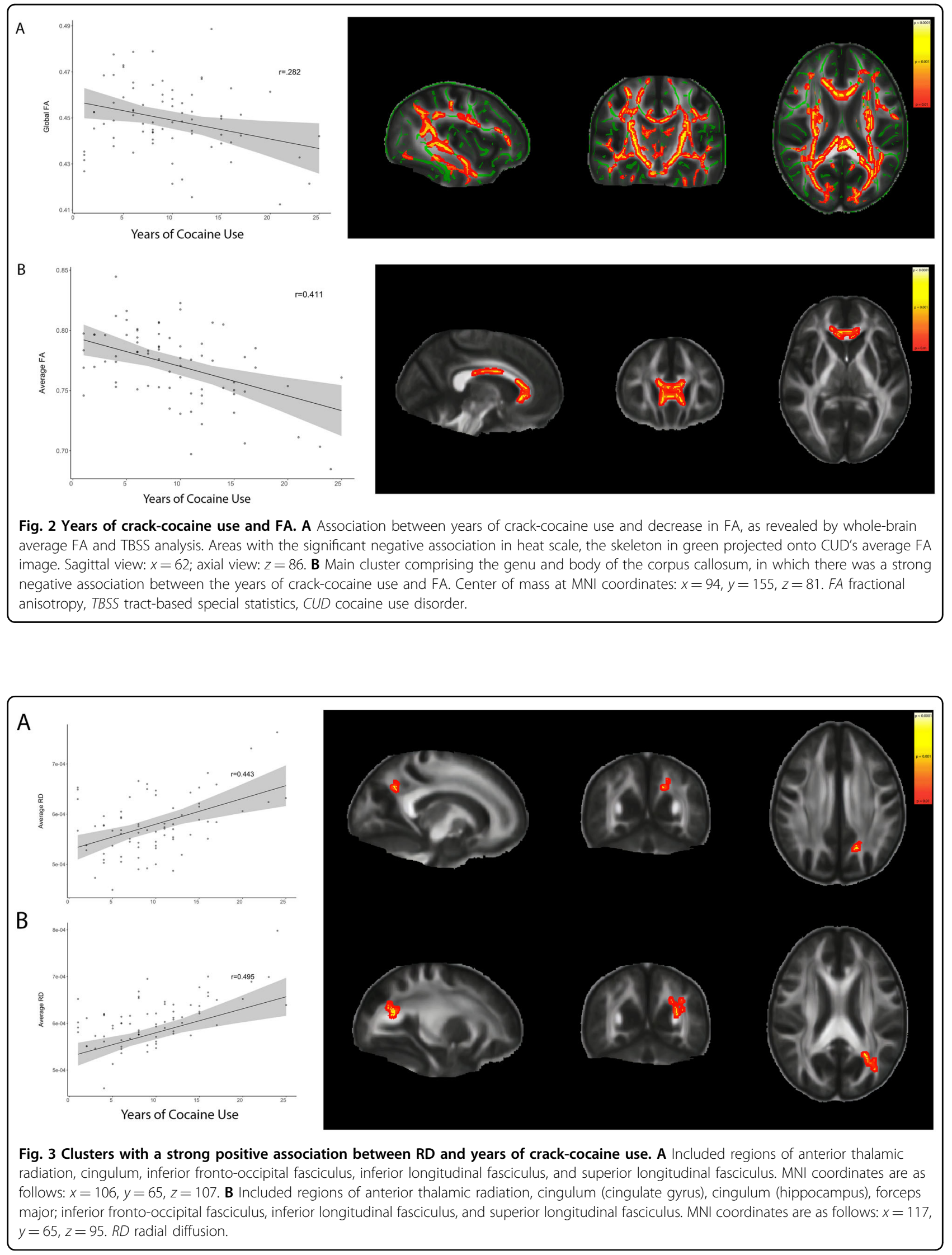


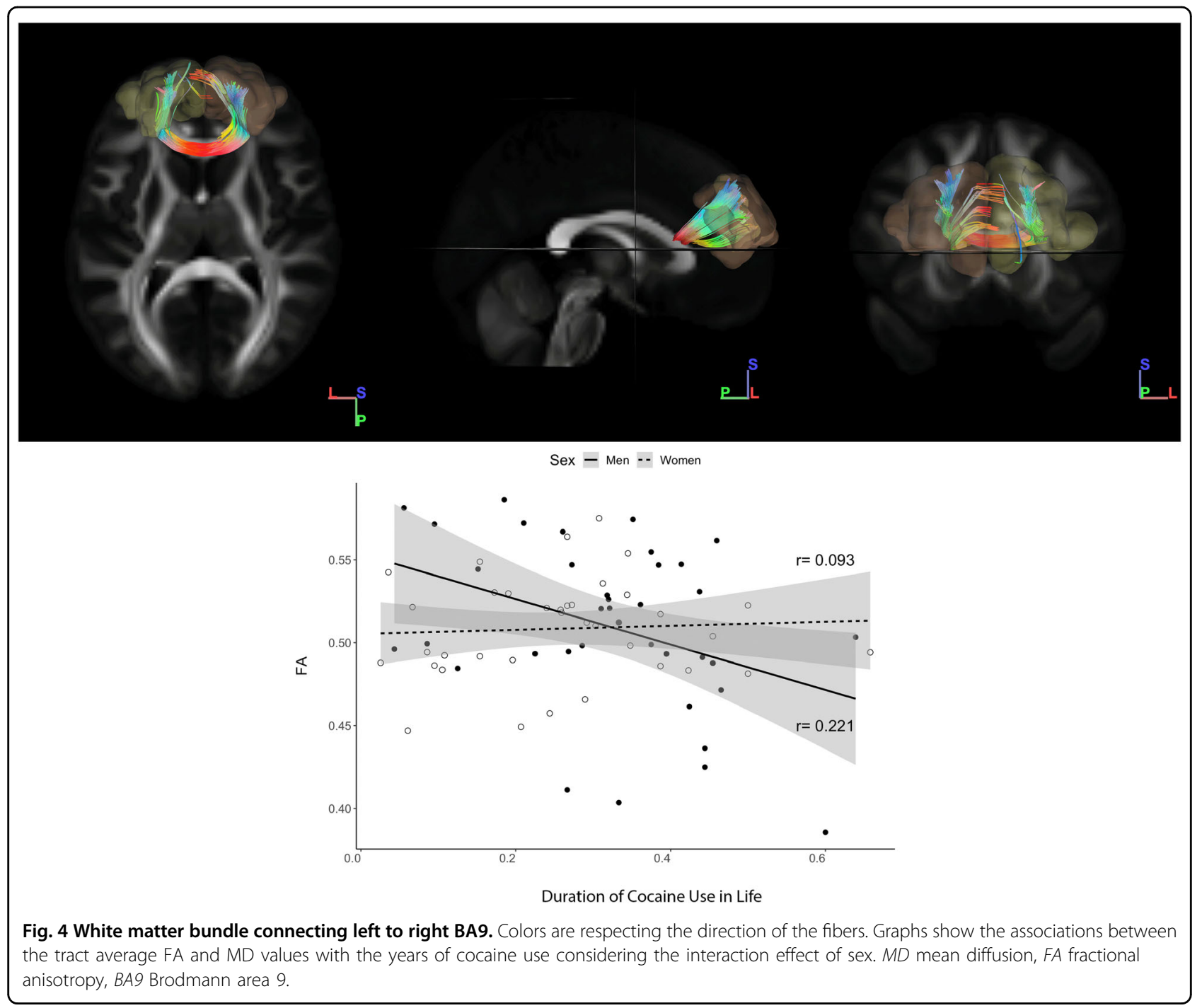

cocaine consumption $(p<0.001 ; t=4.073)$ and alcohol use $(p=0.028 ; t=2.239)$. The cluster displayed in Fig. 3B was only related $\left(F(6,68)=6.587 ; p<0.05\right.$; adjusted $R^{2}=$ $0.319)$ with years of CU $(p<0.001 ; t=4.699)$.

\section{Tractography of prefrontal fibers}

Concerning the fibers connecting the left-BA9 to the right BA9, the CUD group presented lower FA (mean, $0.501 \pm 0.041$ vs. mean, $0.526 \pm 0.030 ; p=0.006)$ and higher RD (mean, $0.630 \pm 0.065$ vs. mean, $0.599 \pm 0.046$; $p<0.001)$ values compared with the $\mathrm{HC}$ group. Regarding the fibers connecting the left-BA9 to the left-BA8, the FA values were lower in the CUD group than in the $\mathrm{HC}$ group (mean, $0.385 \pm 0.027$ vs. mean, $0.395 \pm 0.029 ; p=$ 0.026). Moreover, we found lower FA (mean, $0.405 \pm$ 0.027 vs. mean, $0.414 \pm 0.026 ; p=0.046$ ) and higher RD (mean, $0.631 \pm 0.038$ vs. mean, $0.6179 \pm 0.0338$; $p=0.044$ ) values in the CUD group when comparing tracts connecting the right BA9 to the right BA8.

\section{Tractography clinical associations}

An interaction effect of years of crack-CU and sex within the CUD group was observed on WM tracts connecting the left-BA9 to the right BA9 for FA $(F(5,69)=$ $2.51 ; p=0.037$; adjusted $\left.R^{2}=0.093\right)$. Specifically, we found a negative association between the CU duration in life and FA values in this tract, only in the male users $(t=-2.394 ; p=0.019$; Fig. 4).

\section{Proteomic analysis results}

Based on the effect of the cocaine consumption history on the prefrontal fibers, and concerning the evidence obtained from previous studies regarding the alterations in WM integrity in the prefrontal cortex ${ }^{18,45}$, proteomic 


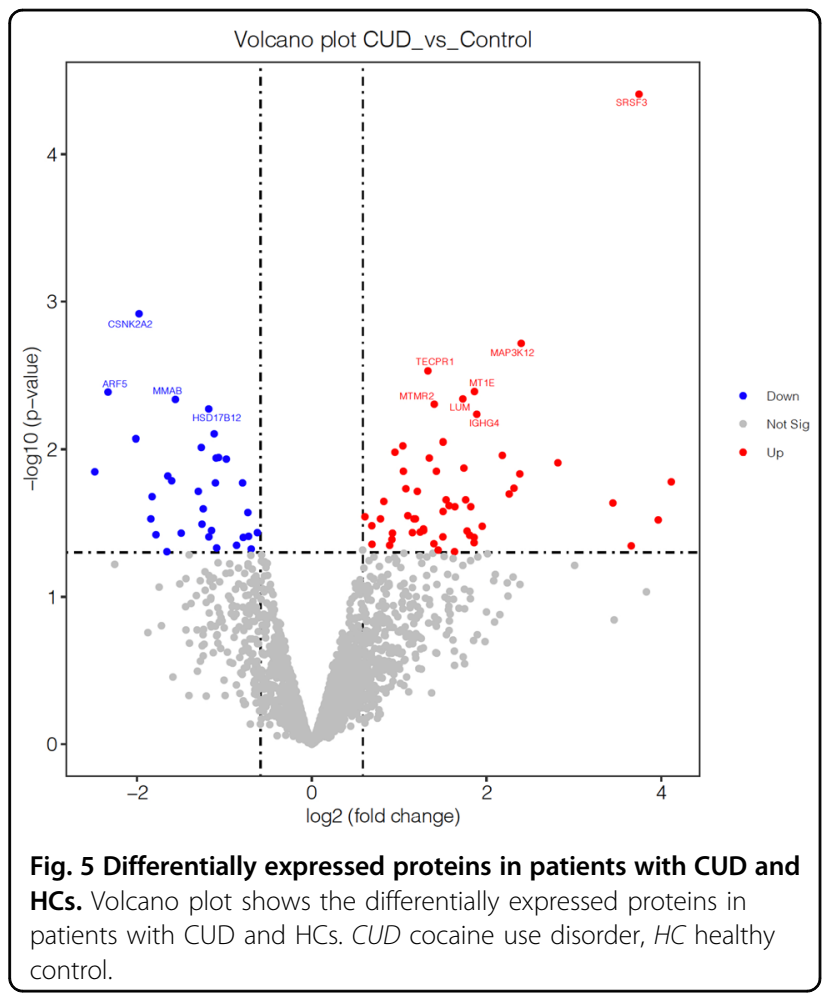

analyses were performed in the BA9 postmortem brain tissue. We used two sets of criteria to define significantly differentially expressed proteins, based on the raw and the adjusted $p$ value, respectively. In both scenarios, we combined the $p$ value threshold with $\log 2$ (FC). By setting the threshold as a $p$ value $<0.05$ and $|\log \mathrm{FC}|>\log 2(1.5)$, we identified 87 proteins that were significantly differentially expressed between the CUD and the HC group. The 87 proteins were used for the enrichment analysis. Pathways related to secretory vesicle formation and trafficking, focal adhesion, and cell-substrate junction were enriched in the CUD group (Supplementary Table 2), in line with previous human myelin proteome analysis findings ${ }^{48}$.

Further analysis with a threshold of $p<0.05$ and $|\operatorname{logFC}|$ $>\log 2(1.5)$ reduced the number of significantly differentially expressed proteins to 11 (Supplementary Table 3). As depicted in the volcano plot (Fig. 5), in the CUD group, we found reduced levels in the following proteins: casein kinase 2 subunit alpha serine-threonine kinase, involved in controlling synapse organization and stability by regulation of NMDA subunits ${ }^{49}$; ADP Ribosylation Factor 5 (ARF5), an activator of phospholipase $\mathrm{D}^{50}$, which is critically involved in myelin development ${ }^{51}$; and Metabolism of Cobalamin Associated B (MMAB) that catalyzes vitamin $B 12^{52}$, which is known to have a key role in myelin repair and maintenance ${ }^{53}$. In addition, among the proteins with higher expression in CUD are serine and arginine rich splicing factor 3 (SRSF3), involved in regulating innate immune responses in activated microglia ${ }^{54}$;
Lumican (LUM), which has an important role in innate immunity $^{55}$; and Myotubularin-related protein 2 (MTMR2), a protein involved in membrane trafficking via dephosphorylation of phosphoinositides, such as PI(3)P. The mass spectrometry proteomics data have been deposited to the ProteomeXchange Consortium via the PRIDE partner repository with the data set identifier PXD025269.

\section{Discussion}

In this study, we showed that crack-cocaine users presented globally distributed alterations in DTI measures (lower FA, higher MD, and RD values) compared with HCs. Moreover, we found that the FA values decreased with increasing years of crack-CU and that this association is particularly strong in a large cluster in the genu and body of the corpus callosum. This area is responsible for the interhemispheric connection of the prefrontal cortex. By reconstructing the specific WM bundles passing through this cluster, connecting both BA9 hemispheres, we found an interaction effect, in which male users with longer CU histories had lower FA and higher RD values. Finally, we found that altered levels of proteins are important for WM integrity and neuroinflammation in the dorsolateral prefrontal cortex BA9 of postmortem brains of patients with CUD. The latter supported the notion that altered DTI metrics maybe because of the impaired WM integrity, perhaps caused by alterations in synapse organization and stability and by vesicular trafficking and, therefore, they are not just a result of nonpathological microstructural disarrangements in the WM, which would also cause altered FA values ${ }^{10}$.

Regarding the development of substance use disorders, altered prefrontal cortex structure/functioning has been implicated in the transition from goal-directed to habitual drug-seeking behavior, and finally to compulsive patterns of drug us ${ }^{56}$. In this sense, although some previous DTI studies have indicated an association between CUD and altered WM integrity in the frontal areas and in the anterior corpus callosum ${ }^{19,57}$, our findings are novel, as we showed that chronic smoked $\mathrm{CU}$ is progressively implicated in decreasing the WM in intrahemispheric connections of the BA9.

We also found two smaller clusters spanning through the dorsal cingulum and posterior thalamic radiation, which presented a strong positive association between RD and years of CU. The cingulum has an important role in connecting the structures of the limbic system, such as the hippocampus and cingulate gyrus. A previous study has supported its role in executive control, emotion, pain, and episodic memory. Especially, the association of pain and episodic memory with the dorsal cingulum and many DTI alterations have been reported in these regions in patients with psychiatric and neurological disorders ${ }^{58}$. RD 
increases in parietal regions were shown to be associated with poor decision making in patients with CUD ${ }^{20}$.

To our knowledge, this was the first DTI study that reported significant whole-brain differences between patients with CUD and HCs concerning FA, RD, and MD. These results reflected a widespread effect of cocaine dependence in the brain, in contrast to the more regionspecific effects found in previous studies ${ }^{59,60}$. This might be because several previous studies have focused on snorted cocaine with less attention being paid to polysubstance use, which ultimately may result in participants with less-severe substance use disorder-induced toxicity. This idea is corroborated by findings showing that altered global FA, MD, and RD were associated with numerous additionally abused substances in patients with $\mathrm{CUD}^{45}$. Global FA and RD values were also associated with years of CU in patients with comorbid CUD and alcohol use disorder ${ }^{18}$. Given that the majority of cocaine users may use and abuse additional drugs ${ }^{61}$, including polysubstance drugs, the inclusion of patients with CUD should allow better generalization of the current findings to real-world settings.

Moreover, previous studies have not specified or not focused on users of smoked cocaine. However, the route of administration and/or the presence of adulterants ${ }^{62}$ related to "crack" cocaine could play an important role in the neurotoxic effect of the drug, as it presents different and more detrimental consequences in the organism ${ }^{63}$. These different effects reflect on clinical measures, where snorted users may achieve better treatment outcomes and better neuropsychological profile than smokers ${ }^{63}$, particularly in prefrontal cortex-dependent tasks ${ }^{64}$.

In this study, we found an interaction between the length of $\mathrm{CU}$ in life and sex. We found that higher $\mathrm{CU}$ duration in life was associated with lower FA values in male participants. This was an interesting result, as studies have shown strong sex differences regarding clinical patterns of substance use disorders, especially in CUD ${ }^{33}$. Men outnumber women in a proportion of 3:1 in CUD prevalence ${ }^{65}$; nevertheless, women are more likely to develop CUD after the first use ${ }^{66}$, progress faster from recreational to pathological use ${ }^{67}$, relapse after treatment ${ }^{68}$, and present a more severe $\mathrm{CUD}^{33}$. Many studies on DTI investigated one sex only ${ }^{15,45}$ or had an uneven distribution of sexes ${ }^{12,60,69}$, which might have masked any possible sex interaction.

Although in our study, there was a significant association between lifelong $\mathrm{CU}$ duration and FA in the tract connecting the BA9s, women had lower global FA values than men in both the CUD and HC groups, except for in the inter-BA9 tract. There are well-documented sexual dimorphisms in DTI studies of healthy participants, the direction of this effect is somewhat conflicting. Nevertheless, a review of the effect of sex on brain connectivity reported that women had overall lower FA values than men $^{70}$. Regarding our findings, this sex effect was also present when analyzing the TBSS AD results, with women also showing lower global AD values. As FA is a compound metric, which relies on the diffusion ratio along the main axis with respect to the diffusion as a whole, there may be multiple paths that mediate the decrease in FA. There could be an increase in $\lambda 2$ and $\lambda 3$ (i.e., higher diffusion perpendicular to the main axis, interpreted as $\mathrm{RD}$ ), or a decrease in $\lambda 1$ (i.e., lower diffusion in the main axis, interpreted as $\mathrm{AD}$ ). Increases in $\mathrm{RD}^{9}$ have been consistently reported as a marker for demyelination and decreases in $\mathrm{AD}$ are not well established in the literature, the precise neurobiological interpretation of FA, RD, and $\mathrm{MD}$ is hard to establish in areas of crossing fibers ${ }^{71}$. In this study, FA differences owing to sex effects regardless of the group were usually accompanied by a decrease in $\mathrm{AD}$ in women; in line, the decrease in FA because of CUD was usually accompanied by an increase in RD, suggesting in vivo evidence of WM deficits associated with CU.

We also found ex vivo evidences of WM deficits associated with CUD. Our postmortem brain proteomics approach revealed evidence of WM deficits and neuroinflammation associated with CUD. To our knowledge, our study was the first that performed an in-depth proteomic analysis in brain samples of participants with CUD, and the first to report a decrease in myelinationrelated proteins in the prefrontal cortex. Interestingly, we found significantly decreased levels of ARF5 (i.e., an activator of phospholipase D) ${ }^{50}$. Specifically, phospholipase D4 has an important role in the mechanisms of myelin development ${ }^{51}$. Furthermore, ARF5 was identified as part of the myelin proteome in the central and peripheral nervous systems ${ }^{72,73}$, suggesting that low ARF5 levels have implications for WM integrity. Reduced levels of MMAB were also found in the CUD group. MMAB has a key role in vitamin B12 metabolism, whereas vitamin B12 deficiencies are critically associated with myelinrelated disorders, such as multiple sclerosis ${ }^{74}$. Vitamin $\mathrm{B} 12$ is also required to produce methionine, which is necessary for methylation reactions that are essential for myelin maintenance and repair ${ }^{53}$. Evidence suggests that reduced vitamin $\mathrm{B} 12$ and methionine levels result in demyelination $^{75}$. In contrast, we detected an increase in the MTMR2 levels in CUD brains, which could be related to a compensatory mechanism. Disruption of MTMR2 is associated with myelin abnormalities, as it may regulate the membrane transport, which is crucially important in the neurons and Schwann cells ${ }^{76}$.

We also observed reduced levels of CSNK2A2, which is part of the kinase CK2 group of proteins involved in neurodevelopment and synaptic plasticity ${ }^{77}$. However, more recently, CSNK2A2 was found to regulate neuroimmune responses through Th17 cell infiltration into 
the brain in a rodent model of autoimmune encephalomyelitis ${ }^{78}$. This is relevant considering the upregulation of LUM and SRSF3 found in the CUD group. Increased LUM levels are associated with Th17 cell activation and inflammation ${ }^{79}$. Moreover, activated microglia cells highly express SRSF3 to regulate inflammatory gene expression and translation ${ }^{54}$. SRSF3 has several binding sites to transcripts of upregulated innate immune genes. This suggests that an imbalance in the levels of immune mediators, such as CSNK2A2, LUM, and SRSF3, could lead to neuroinflammatory signaling cascade in the prefrontal cortex. Given that neuroinflammation is a primary hallmark in several myelin-related disorders and since a misguided inflammatory response is often implicated in demyelination $^{80}$, we found evidence that supported the association of these conditions with chronic substance use throughout life.

These findings from our proteomics analysis could corroborate the observed increase in RD, as a marker of demyelination. A previous postmortem brain study found a decrease in the expression of myelin-related genes in the nucleus accumbens of cocaine abusers ${ }^{81}$. Interestingly, the authors suggested a dysregulation of myelination in these participants. Finally, the negative association between FA and age was also accompanied by an increase in $\mathrm{RD}$ and no change in $\mathrm{AD}$, indicating that the mechanism through which FA decreases in patients with CUD is more related to aging compared with the effect of sex.

Nevertheless, this study had some limitations that should be acknowledged. First, the study design did not allow us to infer whether the DWI alterations are the cause or consequence of CUD. Second, although the DTI and postmortem findings indicate cognitive disruptions that should be reflected in impaired decision-making, affective control, and impulsivity, the present results do not include assessments of psychometric or cognitive/ laboratory tests of these constructs. Additional studies would help confirm these hypotheses by including representative assessments in the test batteries and examining their associations with neuroimaging and histology outcomes. In addition, DWI metrics such as FA, $\mathrm{RD}$, and $\mathrm{MD}$ are dependent on multiple uncontrolled factors, such as axonal coherence, membrane permeability, and fiber orientation ${ }^{10}$ and are especially challenging to analyze in crossing fibers regions, which comprises one-third of the brain ${ }^{71}$. Changes in these metrics must be interpreted carefully, and must not be acknowledged as a synonym of changes in "WM integrity". Finally, despite using two different approaches (in vivo brain imaging and ex vivo brain proteomic analysis) converging to the idea of WM impairment in CUD, we acknowledge that one study assessed data from smoked cocaine users in southern Brazil, whereas the other included both smoked and snorted cocaine users from Houston, TX, USA. Therefore, we could not rule out potential cultural, social, and even ancestry effects in this study.

Despite these limitations, we believe that we demonstrated convergent evidence from in vivo DTI findings and ex vivo proteomic analysis of WM impairments in patients with CUD. Considering that CUD treatment usually has poor outcomes and high relapse rates ${ }^{82}$, such findings bring new insights into sex differences and their pathophysiology.

\section{Acknowledgements}

This study was supported by NIDA and the Fogarty Foundation (RGO, CWB, JMS) (R01DA044859) and by CNPq (RGO) (466802/2014-5) and SENAD (822647/2015). Z.Z. was partially supported by NIH (R01LM012806). The University of Texas System provided funding for the Neuropsychiatric Proteome Database, for which proteomics data from brain tissue was generated by the Mass Spectrometry Core at the University of Texas Medical Branch.

\section{Author details \\ 'Developmental Cognitive Neuroscience Lab (DCNL), Brain Institute, Pontifical Catholic University of Rio Grande do Sul (PUCRS), Porto Alegre, Brazil. ${ }^{2}$ Louis A. Faillace, MD, Department of Psychiatry and Behavioral Sciences, University of Texas Health Science Center at Houston, Houston, TX, USA. ${ }^{3}$ Department of Psychiatry, University of Vermont, Burlington, VT, USA. ${ }^{4}$ Nathan Kline Institute and Child Mind Institute, New York, NY, USA. ${ }^{5}$ Center for Precision Health, School of Biomedical Informatics, University of Texas Health Science Center at Houston, Houston, TX, USA. 'Translational Neuropsychiatry Unit, Department of Clinical Medicine, Aarhus University, Aarhus, Denmark}

\section{Conflict of interest}

The authors declare no competing interests.

\section{Publisher's note}

Springer Nature remains neutral with regard to jurisdictional claims in published maps and institutional affiliations.

Supplementary information The online version contains supplementary material available at https://doi.org/10.1038/s41398-021-01367-x.

Received: 10 March 2021 Revised: 18 March 2021 Accepted: 12 April 2021 Published online: 29 April 2021

\footnotetext{
References

1. Grant, B. F. et al. Epidemiology of DSM-5 drug use disorder: results from the national epidemiologic survey on alcohol and related conditions-III. JAMA Psychiatry 73, 39-47 (2016).

2. United Nations P. World Drug Report 2019 Booklet 4 (2019).

3. Degenhardt, L. et al. Mortality among cocaine users: a systematic review of cohort studies. Drug Alcohol Depend. 113, 88-95 (2011).

4. Dias, A. C. et al. Mortality rate among crack/cocaine-dependent patients: a 12year prospective cohort study conducted in Brazil. J. Subst. Abus. Treat. 41, 273-278 (2011).

5. Ribeiro, M., Dunn, J., Sesso, R., Dias, A. C. \& Laranjeira, R. Causes of death among crack cocaine users. Braz. J. Psychiatry 28, 196-202 (2006).

6. Goldstein, R. Z. \& Volkow, N. D. Drug addiction and its underlying neurobiological basis: neuroimaging evidence for the involvement of the frontal cortex. Am. J. Psychiatry 159, 1642-1652 (2002).

7. Hampton, W. H., Hanik, I. M. \& Olson, I. R. Substance abuse and white matter: findings, limitations, and future of diffusion tensor imaging research. Drug Alcohol Depend. 197, 288-298 (2019).

8. Baliyan, V., Das, C. J., Sharma, R. \& Gupta, A. K. Diffusion weighted imaging: technique and applications. World J. Radiol. 8, 785-798 (2016).
} 
9. Chang, E. H. et al. The role of myelination in measures of white matter integrity: combination of diffusion tensor imaging and two-photon microscopy of CLARITY intact brains. Neuroimage 147, 253-261 (2017).

10. Jones, D. K., Knosche, T. R. \& Turner, R. White matter integrity, fiber count, and other fallacies: the do's and don'ts of diffusion MRI. Neuroimage 73, 239-254 (2013).

11. Huisman, T. A. Diffusion-weighted and diffusion tensor imaging of the brain, made easy. Cancer Imaging 10, S163-S171 (2010).

12. Bell, R. P., Foxe, J. J., Nierenberg, J., Hoptman, M. J. \& Garavan, H. Assessing white matter integrity as a function of abstinence duration in former cocainedependent individuals. Drug Alcohol Depend. 114, 159-168 (2011).

13. He, Q., Li, D., Turel, O., Bechara, A. \& Hser, Y. I. White matter integrity alternations associated with cocaine dependence and long-term abstinence: preliminary findings. Behav. Brain Res 379, 112388 (2020).

14. $\mathrm{Ma}$, L. et al. A preliminary longitudinal study of white matter alteration in cocaine use disorder subjects. Drug Alcohol Depend. 173, 39-46 (2017).

15. van Son, D., Wiers, R. W., Catena, A., Perez-Garcia, M. \& Verdejo-Garcia, A. White matter disruptions in male cocaine polysubstance users: Associations with severity of drug use and duration of abstinence. Drug Alcohol Depend. 168, 247-254 (2016)

16. Beard, C. L. et al. Regional differences in white matter integrity in stimulant use disorders: a meta-analysis of diffusion tensor imaging studies. Drug Alcohol Depend. 201, 29-37 (2019).

17. Suchting R. et al. A meta-analysis of tract-based spatial statistics studies examining white matter integrity in cocaine use disorder. Addict. Biol. 26, e12902 (2020)

18. Tannous, J. et al. Measures of possible allostatic load in comorbid cocaine and alcohol use disorder: brain white matter integrity, telomere length, and antisaccade performance. PLOS ONE 14, e0199729 (2019).

19. $\mathrm{Xu}, \mathrm{J}$. et al. White matter integrity is associated with treatment outcome measures in cocaine dependence. Neuropsychopharmacology 35, 1541-1549 (2010).

20. Lane, S. D. et al. Diffusion tensor imaging and decision making in cocaine dependence. PLOS ONE 5, e11591 (2010).

21. Tang, V. M. et al. White matter deficits assessed by diffusion tensor imaging and cognitive dysfunction in psychostimulant users with comorbid human immunodeficiency virus infection. BMC Res. Notes 8 $515(2015)$

22. Gossop, M., Manning, V. \& Ridge, G. Concurrent use of alcohol and cocaine: differences in patterns of use and problems among users of crack cocaine and cocaine powder. Alcohol Alcohol 41, 121-125 (2006).

23. Hedden, S. L., Malcolm, R. J. \& Latimer, W. W. Differences between adult nondrug users versus alcohol, cocaine and concurrent alcohol and cocaine problem users. Addict. Behav. 34, 323-326 (2009).

24. Viola, T. W. et al. Association between recent cannabis consumption and withdrawal-related symptoms during early abstinence among females with smoked cocaine use disorder. J. Addict. Med. 14, e37-e43 (2020).

25. Viola, T. W. et al. Long-term cannabis abuse and early-onset cannabis use increase the severity of cocaine withdrawal during detoxification and rehospitalization rates due to cocaine dependence. Drug Alcohol Depend. 144, 153-159 (2014).

26. Alia-Klein, N. et al. Gene $x$ disease interaction on orbitofrontal gray matter in cocaine addiction. Arch. Gen. Psychiatry 68, 283-294 (2011).

27. Narayana, P. A. et al. Diffusion tensor imaging of cocaine-treated rodents. Psychiatry Res. 171, 242-251 (2009).

28. Narayana, P. A. et al. Chronic cocaine administration causes extensive white matter damage in brain: diffusion tensor imaging and immunohistochemistry studies. Psychiatry Res. 221, 220-230 (2014).

29. Bannon, M., Kapatos, G. \& Albertson, D. Gene expression profiling in the brains of human cocaine abusers. Addict. Biol. 10, 119-126 (2005).

30. Kristiansen, L. V., Bannon, M. J. \& Meador-Woodruff, J. H. Expression of transcripts for myelin related genes in postmortem brain from cocaine abusers. Neurochem. Res. 34, 46-54 (2009).

31. Kuchling, J. et al. Comparison of probabilistic tractography and tract-based spatial statistics for assessing optic radiation damage in patients with autoimmune inflammatory disorders of the central nervous system. Neuroimage Clin. 19, 538-550 (2018).

32. Miguel, A. Q. C. et al. Sociodemographic characteristics, patterns of crack use, concomitant substance use disorders, and psychiatric symptomatology in treatment-seeking crack-dependent individuals in Brazil. J. Psychoact. Drugs 50 367-372 (2018).
33. Sanvicente-Vieira, B. et al. Sex-based differences in multidimensional clinical assessments of early-abstinence crack cocaine users. PLOS ONE 14, e0218334 (2019).

34. First M., Williams J., Spitzer R., Karg R. Scid-5-C V - Versão Clínica. 5th edn Artmed (2017).

35. Wechsler D. Wechsler Abbreviated Scale of Intelligence-Second Edition (WASI-II). (NCS Pearson, 2011).

36. Cacciola, J. S., Alterman, A. I., Habing, B. \& McLellan, A. T. Recent status scores for version 6 of the Addiction Severity Index (ASI-6). Addiction 106, 1588-1602 (2011).

37. Andersson, J. L. R. \& Sotiropoulos, S. N. An integrated approach to correction for off-resonance effects and subject movement in diffusion MR imaging. Neuroimage 125, 1063-1078 (2016).

38. Smith, S. M. \& Nichols, T. E. Threshold-free cluster enhancement: addressing problems of smoothing, threshold dependence and localisation in cluster inference. Neuroimage 44, 83-98 (2009).

39. Bach, M. et al. Methodological considerations on tract-based spatial statistics (TBSS). Neuroimage 100, 358-369 (2014).

40. Kodiweera, C., Alexander, A. L., Harezlak, J., McAllister, T. W. \& Wu, Y. C. Age effects and sex differences in human brain white matter of young to middleaged adults: a DTI, NODDI, and q-space study. Neuroimage 128, 180-192 (2016).

41. van der Holst, H. M. et al. Microstructural integrity of the cingulum is related to verbal memory performance in elderly with cerebral small vessel disease: the RUN DMC study. Neuroimage 65, 416-423 (2013).

42. van Norden, A. G. et al. Diffusion tensor imaging of the hippocampus and verbal memory performance: the RUN DMC study. Hum. Brain Mapp. 33, 542-551 (2012).

43. Ozalay, O. et al. The relationship between the anterior corpus callosum size and prefrontal cortex volume in drug-free depressed patients. J. Affect Disord. 146, 281-285 (2013).

44. Putnam, M. C., Wig, G. S., Grafton, S. T., Kelley, W. M. \& Gazzaniga, M. S. Structural organization of the corpus callosum predicts the extent and impact of cortical activity in the nondominant hemisphere. J. Neurosci. 28, 2912-2918 (2008).

45. Kaag, A. M. et al. White matter alterations in cocaine users are negatively related to the number of additionally (ab)used substances. Addict. Biol. 22, 1048-1056 (2017)

46. Li, Z. et al. Prenatal cocaine exposure alters functional activation in the ventral prefrontal cortex and its structural connectivity with the amygdala. Psychiatry Res. 213, 47-55 (2013).

47. Petrides, M., Tomaiuolo, F., Yeterian, E. H. \& Pandya, D. N. The prefrontal cortex: comparative architectonic organization in the human and the macaque monkey brains. Cortex 48, 46-57 (2012).

48. Ishii, A. et al. Human myelin proteome and comparative analysis with mouse myelin. Proc. Natl Acad. Sci. USA 106, 14605-14610 (2009).

49. Kimura, R. \& Matsuki, N. Protein kinase CK2 modulates synaptic plasticity by modification of synaptic NMDA receptors in the hippocampus. J. Physiol. 586, 3195-3206 (2008)

50. Bruntz, R. C., Lindsley, C. W. \& Brown, H. A. Phospholipase D signaling pathways and phosphatidic acid as therapeutic targets in cancer. Pharm. Rev. 66, 1033-1079 (2014)

51. Chiba, T. et al. Microglial phospholipase D4 deficiency influences myelination during brain development. Proc. Jpn. Acad. Ser. B Phys. Biol. Sci. 92, 237-254 (2016).

52. Dobson, C. M. et al. Identification of the gene responsible for the cblB complementation group of vitamin B12-dependent methylmalonic aciduria. Hum. Mol. Genet 11, 3361-3369 (2002).

53. Miller, A., Korem, M., Almog, R. \& Galboiz, Y. Vitamin B12, demyelination, remyelination and repair in multiple sclerosis. J. Neurol. Sci. 233, $93-97$ (2005).

54. Boutej, H. et al. Diverging mRNA and protein networks in activated microglia reveal SRSF3 suppresses translation of highly upregulated innate immune transcripts. Cell Rep. 21, 3220-3233 (2017).

55. Hayashi, Y. et al. Lumican is required for neutrophil extravasation following corneal injury and wound healing. J. Cell Sci. 123, 2987-2995 (2010).

56. Everitt, B. J. \& Robbins, T. W. Drug addiction: updating actions to habits to compulsions ten years on. Annu Rev. Psychol. 67, 23-50 (2016).

57. Moeller, F. G. et al. Reduced anterior corpus callosum white matter integrity is related to increased impulsivity and reduced discriminability in cocainedependent subjects: diffusion tensor imaging. Neuropsychopharmacology $\mathbf{3 0}$, 610-617 (2005). 
58. Bubb, E. J., Metzler-Baddeley, C. \& Aggleton, J. P. The cingulum bundle: anatomy, function, and dysfunction. Neurosci. Biobehav Rev. 92, 104-127 (2018).

59. Lim, K. O. et al. Brain macrostructural and microstructural abnormalities in cocaine dependence. Drug Alcohol Depend. 92, 164-172 (2008).

60. Vaquero, L. et al. Cocaine addiction is associated with abnormal prefrontal function, increased striatal connectivity and sensitivity to monetary incentives, and decreased connectivity outside the human reward circuit. Addict. Biol. 22, 844-856 (2017)

61. Liu, Y., Williamson, V., Setlow, B., Cottler, L. B. \& Knackstedt, L. A. The importance of considering polysubstance use: lessons from cocaine research. Drug Alcohol Depend. 192, 16-28 (2018).

62. Ribeiro, M. et al. Adulterants in crack cocaine in Brazil. Trends Psychiatry Psychother. 41, 186-190 (2019).

63. Kiluk, B. D., Babuscio, T. A., Nich, C. \& Carroll, K. M. Smokers versus snorters: do treatment outcomes differ according to route of cocaine administration? Exp. Clin. Psychopharmacol. 21, 490-498 (2013).

64. Oliveira, H. P. et al. The route of administration exacerbates prefrontal functional impairments in crack cocaine users. Psychol. Addict. Behav. 32, 812-820 (2018).

65. Winstock A., Barrat M., Ferris J., Maier L. Global Drug Survey. GDS Core Research Team (2017).

66. Elman, I., Karlsgodt, K. H. \& Gastfriend, D. R. Gender differences in cocaine craving among non-treatment-seeking individuals with cocaine dependence. Am. J. Drug Alcohol Abus. 27, 193-202 (2001).

67. Zilberman, M. L., Hochgraf, P. B. \& Andrade, A. G. Gender differences in treatment-seeking Brazilian drug-dependent individuals. Subst. Abus 24, 17-25 (2003).

68. Fattore, L. \& Melis, M. Sex differences in impulsive and compulsive behaviors: a focus on drug addiction. Addict. Biol. 21, 1043-1051 (2016).

69. Ersche, K. D. et al. Abnormal brain structure implicated in stimulant drug addiction. Science 335, 601-604 (2012).
70. Gong, G., He, Y. \& Evans, A. C. Brain connectivity: gender makes a difference Neuroscientist 17, 575-591 (2011).

71. Jeurissen, B., Leemans, A., Tournier, J. D., Jones, D. K. \& Sijbers, J. Investigating the prevalence of complex fiber configurations in white matter tissue with diffusion magnetic resonance imaging. Hum. Brain Mapp. 34, 2747-2766 (2013).

72. Siems S. B. et al. Proteome profile of peripheral myelin in healthy mice and in a neuropathy model. Elife 9, e51406 (2020).

73. Jahn, O., Tenzer, S. \& Werner, H. B. Myelin proteomics: molecular anatomy of an insulating sheath. Mol. Neurobiol. 40, 55-72 (2009).

74. Dardiotis, E. et al. Vitamin B12, folate, and homocysteine levels and multiple sclerosis: a meta-analysis. Mult. Scler. Relat. Disord. 17, 190-197 (2017).

75. Nemazannikova, N., Mikkelsen, K., Stojanovska, L., Blatch, G. L. \& Apostolopoulos, V. Is there a link between vitamin B and multiple sclerosis? Med Chem. 14, 170-180 (2018).

76. Bolino, A. et al. Disruption of Mtmr2 produces CMT4B1-like neuropathy with myelin outfolding and impaired spermatogenesis. J. Cell Biol. 167, 711-721 (2004).

77. Lettieri A. et al. Protein kinase CK2 subunits differentially perturb the adhesion and migration of GN11 cells: a model of immature migrating neurons. Int. J. Mol. Sci. 20, 5951 (2019).

78. Jang, S. W. et al. Casein kinase 2 is a critical determinant of the balance of Th17 and Treg cell differentiation. Exp. Mol. Med. 49, e375 (2017).

79. Castillo, E. F. et al. Lumican negatively controls the pathogenicity of murine encephalitic TH17 cells. Eur. J. Immunol. 46, 2852-2861 (2016).

80. McMurran, C. E., Jones, C. A., Fitzgerald, D. C. \& Franklin, R. J. CNS remyelination and the innate immune system. Front. Cell Dev. Biol. 4, 38 (2016).

81. Albertson, D. N. et al. Gene expression profile of the nucleus accumbens of human cocaine abusers: evidence for dysregulation of myelin. J. Neurochem 88, 1211-1219 (2004)

82. Ronsley, C. et al. Treatment of stimulant use disorder: a systematic review of reviews. PLOS ONE 15, e0234809 (2020) 